\title{
Angiodisplasia intestinal em paciente com doença renal crônica: relato de caso
}

\author{
Angiodysplasia in patient with chronic kidney disease: case report
}

Angiodisplasia intestinal en un paciente con enfermedad renal crónica: reporte de caso

Rafaelly Taketomi de Magalhães ${ }^{1 *}$, Ana Wanda Guerra Barreto Marinho', Samanta Samara Bicharra dos Santos ${ }^{1}$, José Lopes Cavalcante Júnior ${ }^{1}$, Larissa Lima do Nascimento ${ }^{1}$, Kátia Batista de Oliveira ${ }^{1}$, Maykon da Silveira Dias' ${ }^{1}$, Daniel Monteiro Queiroz¹, Ana Beatriz da Cruz Lopo de Figueiredo'1.

\section{RESUMO}

Objetivo: Relatar e discutir sobre a Angiodisplasia Intestinal em paciente com Doença Renal Crônica (DRC) em um hospital de atendimento terciário. Detalhamento do caso: Paciente do sexo masculino de 67 anos, diabético, hipertenso e com diagnóstico de Doença Renal Crônica dialítica há 3 anos, evoluindo com sangramento gastrointestinal há 2 anos e meio, epistaxe e perda ponderal. Foi submetido a exame de cápsula endoscópica que evidenciou múltiplas angiectasias ao longo do intestino delgado. Realizado inicialmente terapêutica farmacológica com Talidomida, Octeotidra e Desmopressina e posteriormente tratamento através de Enteroscopia com duplo balão e terapia hemostática com coagulação por plasma de argônio. Considerações finais: A angiodisplasia intestinal é uma das principais causas de hemorragia gastrointestinal nos pacientes com DRC. Sua causa exata permanece desconhecida. O diagnóstico pode ser realizado através de técnicas endoscópicas e o tratamento pode tornar-se necessário em pacientes sintomáticos, através de terapia farmacológica e coagulação com plasma de argônio.

Palavras-chave: Angiodisplasia, Hemorragia gastrointestinal, Doença renal crônica.

\begin{abstract}
Objective: Report and discuss about Intestinal Angiodysplasia in a patient with Chronic Kidney Disease (CKD) in a tertiary care hospital. Case details: 67-year-old male patient, diabetic, hypertensive and diagnosed with dialysis chronic kidney disease for 3 years, evolving with gastrointestinal bleeding for 2 and a half years, epistaxis and weight lisos. He underwent an endoscopic capsule examination that showed multiple angiectasis along the small intestine. Initially, pharmacological therapy was performed with Thalidomide, Octeotidra and Desmopressin and subsequently treated through double balloon Enteroscopy and hemostatic therapy with argon plasma coagulation.

Final considerations: Intestinal angiodysplasia is a major cause of gastrointestinal bleeding in patients with CKD. Its exact cause remains unknown. The diagnosis can be made through endoscopic techniques and treatment may become necessary in symptomatic patients, through pharmacological therapy and clotting with argon plasma.
\end{abstract}

Keywords: Angiodysplasia, Gastrointestinal bleeding, Chronic kidney disease.

1 Universidade Federal do Amazonas (UFAM). Hospital Universitário Getúlio Vargas (HUGV). Manaus - AM.

*E-mail: rafaelly_tm@hotmail.com

SUBMETIDO EM: $2 / 2020 \quad$ ACEITO EM: 3/2020 ～～PUBLICADO EM: 5/2020

REAS/EJCH | Vol.Sup.n.48 | e3190 | DOI: https://doi.org/10.25248/reas.e3190.2020 Página 1 de 6 


\section{RESUMEN}

Objetivo: Informar y debatir sobre la angiodisplasia intestinal en un paciente con enfermedad renal crónica (ERC) en un hospital de atención terciaria. Detalles del caso: Paciente masculino de 67 años, diabético, hipertenso y diagnosticado con enfermedad renal crónica en diálisis durante 3 años, evolucionando con sangrado gastrointestinal durante 2 años y medio, epistaxis y pérdida de peso. Se sometió a un examen de cápsula endoscópica que mostró múltiples angiectasias a lo largo del intestino delgado. Inicialmente, la terapia farmacológica se realizó con Talidomida, Octeotidra y Desmopresina y posteriormente se trató mediante enteroscopia de doble balón y terapia hemostática con coagulación con plasma de argón. Consideraciones finales: La angiodisplasia intestinal es una causa importante de hemorragia gastrointestinal en pacientes con ERC. Su causa exacta sigue siendo desconocida. El diagnóstico puede realizarse mediante técnicas endoscópicas y el tratamiento puede ser necesario en pacientes sintomáticos, mediante terapia farmacológica y coagulación con plasma de argón.

Palabras clave: Angiodisplasia, Sangrado gastrointestinal, Enfermedad renal crónica.

\section{INTRODUÇÃO}

A Angiodisplasia intestinal é uma malformação arteriovenosa de etiologia desconhecida que consiste em pequenos vasos anormais, dilatados e tortuosos, com paredes finas e diâmetro menor que $10 \mathrm{~mm}$ dentro da camada mucosa e submucosa do intestino, frequentemente múltiplas (SAMI SS, et al., 2013). Consiste em um dos diagnósticos diferenciais mais importantes a ser considerado em pacientes com sangramento do trato digestivo de origem obscura e apesar de ser responsável por cerca de 19 a $32 \%$ de todos os sangramentos gastrointestinais baixos (GALANOPOULOS G, 2012) é frequentemente subdiagnosticado devido à maior dificuldade em identificá-lo.

Vasos sanguíneos aberrantes podem estar presentes desde o nascimento ou se desenvolver como parte de síndromes herdadas, mas a grande maioria é observada em idades avançadas. As razões para a distorção das estruturas vasculares observadas com o avanço da idade são pouco conhecidas. $A$ maioria das lesões angiodisplásicas são detectadas em pacientes com mais de 60 anos de idade, embora a apresentação em pacientes com Doença Renal Crônica (DRC) possa ser mais precoce (SAMI SS, et al., 2013). Não existe predileção racial nos casos de angiodisplasia intestinal e ocorre com igual frequência em homens e mulheres (AL-HAMID H, 2019).

As manifestações clínicas da angiodisplasia são variadas. Pode ser encontrada durante a investigação do sangramento gastrointestinal, ou pode ser descoberta incidentalmente durante uma avaliação endoscópica realizada por outros motivos. Se ocorrer sangramento, o sangramento tende a ser recorrente e crônico. No entanto, sangramento agudo acentuado causando hipotensão e instabilidade hemodinâmica, pode ocorrer (SAMI SS, et al., 2013).

A DRC consiste em um fator de risco para sangramento gastrointestinal, estando associada a um risco aumentado de patologias como gastrite, úlcera péptica e angiodisplasia. Estas são importantes causas de anemia refratária à eritropoetina em pacientes sob terapia dialítica. Esses pacientes evoluem com desfechos desfavoráveis o que eleva a sua morbimortalidade se comparada à população geral e a pacientes sem alteração de função renal (MUFTAH M, et al., 2019).

A razão para o aumento da prevalência entre pacientes com DRCainda não está clara. Uma possível explicação é que as lesões são detectadas com mais frequência devido à maior exposição a anticoagulates e ao risco aumentado de sangramento associado à disfunção plaquetária induzida pela uremia, levando a um número maior de exames endoscópicos e consequentemente a uma maior detecção de angiodisplasias (SALTZMAN JR, 2018).

REAS/EJCH | Vol.Sup.n.48 | e3190 | DOI: https://doi.org/10.25248/reas.e3190.2020 Página 2 de 6 
Apresentamos um relato do caso clínico de um paciente portador de DRC em estágio dialítico que foi diagnosticado com angiodisplasia intestinal por meio de cápsula endoscópica e submetido a terapias farmacológicas e à terapia hemostática com plasma de argônio.

\section{RELATO DO CASO}

Paciente do sexo masculino, 67 anos, natural de Tefé-Am, procedente de Manaus - AM. Há 3 anos iniciou terapia dialítica, hemodiálise, como tratamento para Doença Renal Crônica. Foi admitido em janeiro de 2019 na enfermaria de Nefrologia em um hospital universitário para investigação de sangramento gastrointestinal, anemia sintomática, epistaxe ocasional e perda ponderal de 20 quilos. Havia iniciado quadro de melena há 2 anos e meio, com exacerbação há 1 ano e necessidade de constantes idas ao Pronto Socorro para hemotransfusão.

Antecedentes de Diabetes Mellitus há 8 anos, Hipertensão Arterial Sistêmica há 4 anos e Hiperplasia Prostática Benigna há 3 anos. Ex-etilista abstêmio há 3 anos e ex-tabagista com carga de 18 maços-ano, abstêmio há 39 anos. Negava uso de drogas ilícitas. Fazia uso de Losartana (50mg/dia), Omeprazol (60mg/dia), Cloridrato de Sevelamer (2.400 mg/dia) e Dutasterida+Tansulosina (0,5mg+0,4mg/dia).

Realizou Endoscopia Digestiva Alta (EDA) e Colonoscopia durante atendimento em Hospital Pronto Socorro, que evidenciaram sangramento difuso, pontual em mucosa de cólon ascendente e descendente, respectivamente. Após admissão na enfermaria de Nefrologia, foi submetido novamente à investigação com EDA que demonstrou ectasias venosas no terço proximal e médio do esôfago e gastrite enantematosa leve de antro.

Uma nova Colonoscopia evidenciou doença diverticular no cólon direito não complicada, pólipos sésseis no cólon transverso e resíduos hemáticos desde o íleo terminal até o reto, sugerindo sangramento prévio com provável origem no intestino médio. Aventou-se então a hipótese de Angiectasias difusas em intestino delgado e cólon.

Por ser uma região anatômica de difícil acesso para determinação precisa do sítio de sangramento, o paciente foi submetido ao exame endoscópico mediante uso de cápsula endoscópica que demonstrou erosões em corpo gástrico, sangramento ativo em duodeno distal e jejuno proximal, grande quantidade de coágulos aderidos à mucosa e angiectasias ao longo de todo delgado (Figura 1).

Figura 1 - Imagens capturadas através da cápsula endoscópica.
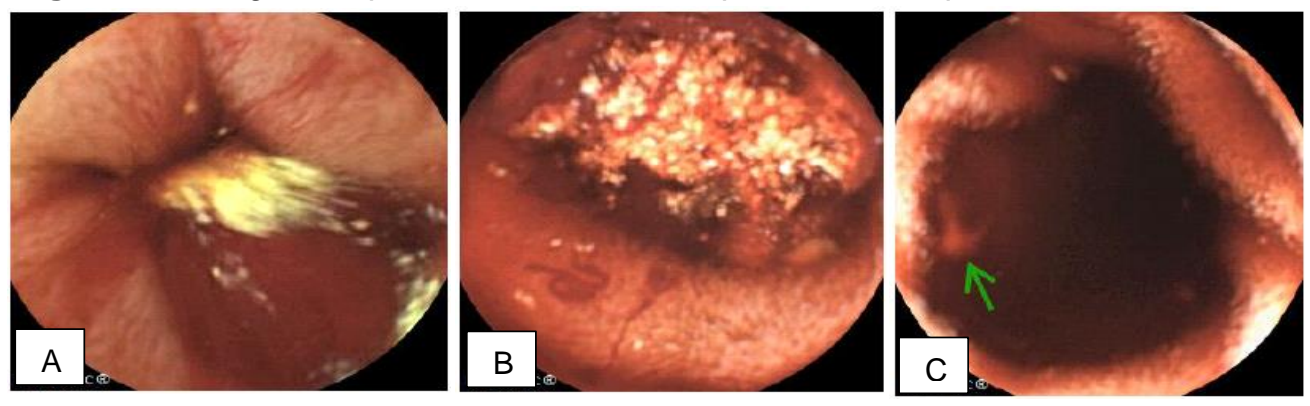

Legenda: Figura 1A: Imagem de sangramento ativo. 1B: Presença de angiectasia. 1C: Sítio de sangramento ativo indicado na seta.

Fonte: Magalhães RT, et al., 2020.

Diante desses achados, o paciente iniciou tratamento com Talidomida 100mg/dia e Desmopressina intranasal. Foi sugerido também tratamento local por enteroscopia com duplo balão, porém não foi possível realizar. $O$ paciente foi submetido à Arteriografia em março de 2019 que também não determinou precisamente o sítio de sangramento.

REAS/EJCH | Vol.Sup.n.48 | e3190 | DOI: https://doi.org/10.25248/reas.e3190.2020 Página 3 de $\mathbf{6}$ 
A enteroscopia com duplo balão foi então realizada em abril de 2019, sendo identificadas múltiplas lesões planas na terceira e quarta porção do duodeno, medindo até $0,4 \mathrm{~cm}$, com coloração vermelho vivo, de limites regulares e precisos. Uma das lesões apresentava coágulo aderido de coloração vermelho vivo, sendo removido e identificado único foco de sangramento ativo e realizado hemostasia por plasma de Argônio das lesões (Figura 2).

Figura 2 - Imagens obtidas a partir da Enteroscopia com duplo balão.

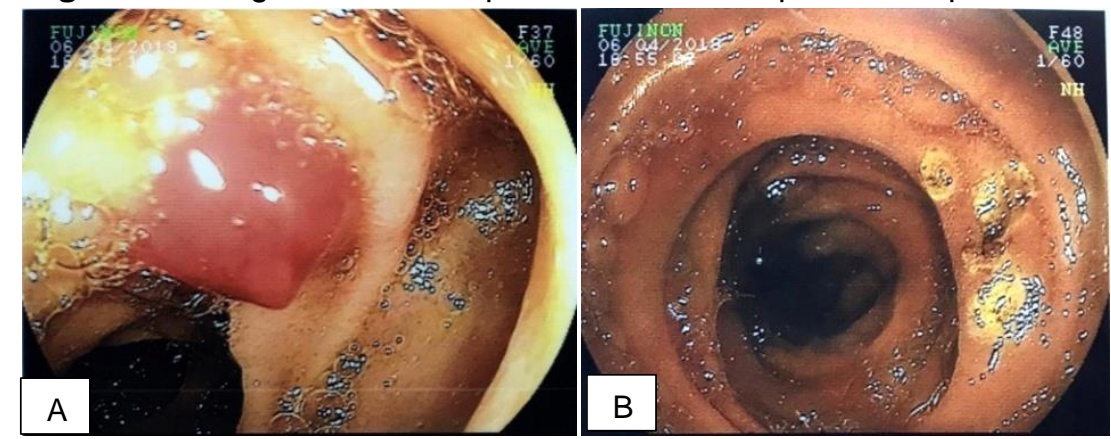

Legenda: Figura 2A: Coágulo aderido em mucosa duodenal. 2B: Após hemostasia por plasma de Argônio das lesões.

Fonte: Magalhães RT, et al., 2020.

O paciente evoluiu com melhora do quadro de melena e necessidade de hemotransfusão por aproximadamente uma semana, mas voltou a apresentar novos episódios de sangramento intestinal, melena e instabilidade hemodinâmica por anemia. Apesar dos cuidados intensivos, cobertura antibiótica, uso de octreotida e transfusão de hemocomponentes paciente faleceu em abril de 2019. Nesta última internação fez uso de 58 concentrados de hemácias num período de 104 dias.

\section{DISCUSSÃO}

Angiodisplasias são as anomalias vasculares mais comumente encontradas no Trato Gastrointestinal (TGI) em pacientes com mais de 60 anos. Sua prevalência como causa de hemorragia nos pacientes com Doença Renal Crônica varia de 19 a 32\% em comparação a 5\% em indivíduos com função renal normal, sendo responsável por até metade dos episódios de sangramento recorrente (ZUCKERMAN GR, et al., 1985). O sangramento pode ser oculto e intermitente, mas ocasionalmente pode manifestar-se como hemorragia maciça (KALMAN RS, 2014).

A etiologia das lesões angiodisplásicas e sua correlação com DRC não são bem estabelecidas, porém existem algumas condições particulares favoráveis ao desenvolvimento desse tipo de lesão. O processo degenerativo vascular na parede intestinal é acelerado pela hipoxemia e uremia em pacientes com DRC. Diversas comorbidades como a microangiopatia diabética e a doença vascular periférica aterosclerótica podem favorecer o comprometimento da microcirculação intestinal que pode causar deformação e, eventualmente, comunicações arteriovenosas (KAAROUD H, et al., 2008).

O metabolismo alterado do cálcio observado na DRC, devido ao hiperparatireoidismo secundário, pode gerar calcificações que comprometem a microcirculação da mucosa intestinal (GALANAPOULOS G, 2012). Pacientes com DRC também têm maior propensão para as perdas de sangue gastrointestinais em comparação com a população em geral, tal fato pode ser atribuído à disfunção plaquetária urêmica, com comprometimento da adesão e agregação plaquetária, e uso de anticoagulantes (BOCCARDO P, et al., 2004). A localização das lesões angiogisplásicas no intestino costuma ser difícil. Inicialmente, os pacientes com sangramento recorrente em TGI

REAS/EJCH | Vol.Sup.n.48 | e3190 | DOI: https://doi.org/10.25248/reas.e3190.2020 Página 4 de 6 
são avaliados por Endoscopia Digestiva Alta ou Colonoscopia, com base nas características da hemorragia. Se tais investigações não revelarem a localização, procede-se à avaliação do intestino delgado (MUFTAH M, et al., 2019).

Apesar dos métodos disponíveis, a utilização da cápsula endoscópica no diagnóstico de doenças do intestino delgado e sua superioridade em relação à radiografia do intestino delgado, enteroscopia, tomografia computadorizada e angiografia foram aceitas na prática clínica (JACKSON CS e STRONG R, 2017).

A cápsula endoscópica permite revelar o local da hemorragia no intestino delgado em casos de insucesso por técnica endoscópica. É considerada investigação de primeira linha preferida para o intestino delgado no contexto de sangramento obscuro do TGI, pois é segura, aceitável e possui rendimento significativamente maior ou pelo menos equivalente quando comparado com outras modalidades mais invasivas, como enteroscopia, angiografia mesentérica e enteroscopia intra-operatória Sami SS, et al. (2014), além de oferecer planejamento de intervenção terapêutica. Em um estudo realizado por Docherty E, et al. (2015) que avaliou o uso de cápsula endoscópica no intestino delgado em pacientes com DRC, as angiectasias foram os achados mais comumente encontrados. $O$ sítio do sangramento encontrado no paciente do caso relatado condiz com os dados da literatura acerca do local mais acometido.

A angiografia também é uma opção diagnóstica que pode ser usada em casos de sangramento ativo a fim de localizar o ponto da hemorragia, através da visualização do extravasamento do agente de contraste no lúmen intestinal (GALANOPOULOS G, 2012). A angiografia é o exame padrão, entretanto, possui um menor rendimento diagnóstico em pacientes com sangramento obscuro quando comparado com o uso de cápsula endoscópica (LEUNG WK, et al., 2012). No caso relatado, a angiografia não obteve sucesso quanto à localização precisa do sítio de sangramento.

O manejo dos pacientes com angiodisplasia depende do contexto clínico em que são diagnosticados. Pacientes assintomáticos cujas lesões são encontradas acidentalmente não necessitam ser submetidos a intervenções terapêuticas, visto que o risco de sangramento futuro nesse subgrupo de pacientes é baixo e a maioria permanece assintomática (FOUTCH PG, 1995). Entretanto, o tratamento pode ser considerado em casos de manifestação clínica e o grau de invasão da abordagem dependerá do tamanho, local e número de lesões (SAMI SS, et al., 2014). As modalidades de tratamento incluem terapias endoscópicas, angiografia com embolização, ressecção cirúrgica e terapia farmacológica (JACKSON CS e STRONG R, 2017).

A terapia endoscópica é considerada terapia inicial eficaz para angiodisplasias, embora as taxas de ressangramento sejam altas, especialmente quando localizadas no intestino delgado (JACKSON CS e STRONG $R$, 2017). Porém, os dados sobre desfechos a longo prazo são controversos e não há estudos avaliando a intervenção endoscópica para sangramento obscuro do TGI em pacientes com DRC.

A enteroscopia com duplo balão é uma técnica endoscópica que detecta pontos de sangramento mesmo em locais de difícil acesso no intestino delgado. Permite realizar coagulação por plasma de argônio e posicionamento de clipes, sendo a coagulação por plasma de argônio o procedimento hemostático mais utilizado (MAY A, et al., 2011).

Consiste em eletrocoagulação sem contato, pela qual a corrente elétrica de alta frequência pode ser fornecida ao tecido através de gás argônio ionizado e possui como vantagem a facilidade de aplicação, tratamento rápido de múltiplas lesões e segurança devido à profundidade reduzida de penetração (OLMOS JA, et al., 2004; SAMI SS, et al., 2014).

Estudos demonstraram que a terapia hormonal teria sido ineficaz para controle de sangramento em pacientes com DRC e dados sugerem que seu efeito como forma de prevenção são controversos e escassos (MUFTAH M, et al., 2019). Devido à eficácia e segurança de outros agentes descritos para a terapêutica da Angiodisplasia optou-se, portanto, por não realizar terapia hormonal no paciente do caso relatado.

REAS/EJCH | Vol.Sup.n.48 | e3190 | DOI: https://doi.org/10.25248/reas.e3190.2020 Página $\mathbf{5}$ de $\mathbf{6}$ 
A Talidomida tem ação imunomoduladora, anti-inflamatória e anti-angiogênicadevido à supressão do Fator de Crescimento Endotelial Vascular (VEGF) que está envolvido na formação de lesões angiectásicas (COSTA TB, et al., 2009). Pode diminuir número, tamanho e intensidade da coloração das angiectasias do intestino delgado (BAUDITZ J, et al. 2004). Por não ter sua farmacocinética bem determinada em pacientes com disfunção renal, cuidados devem ser tomados em pacientes com níveis de creatinina $>300 \mu \mathrm{mol} / \mathrm{L}$ (ou 3,39 mg/dL), pelo risco de ocorrer hipercalemia.Mas em paciente já em nível dialítico o uso da Talidomida pode ser benéfico (MIMIDIS K, et al., 2008).

Octreotida é um análogo das omatostatina que pode ser uma opção eficaz para o tratamento do sangramento por angiodisplasia especialmente naqueles com sangramento recorrente, apesar da intervenção endoscópica. Atua reduzindo o fluxo sanguíneo no território esplâncnico, inibindo a angiogênese, melhorando a agregação plaquetária e aumentando a resistência vascular (MUFTAH M, et al., 2019). A Desmopressina também pode ser utilizada em pacientes com DRC quando o sangramento está relacionado à disfunção plaquetária urêmica. (MUFTAH M, et al., 2019). A terapia farmacológica com Talidomida, Octreotida e Desmopressina não obteve sucesso em cessar o sangramento gastrointestinal do paciente relatado no estudo, sendo necessário o tratamento com coagulação por plasma de argônio.

Apresentamos o relato de um caso de sangramento no intestino delgado por angiodisplasia diagnosticado por exame de cápsula endoscópica em paciente portador de DRC. A angiodisplasia intestinal é uma das principais causas de hemorragia gastrointestinal nos pacientes com DRC. Sua causa exata permanece desconhecida. $O$ diagnóstico pode ser realizado através de técnicas endoscópicas e o tratamento pode tornar-se necessário em pacientes sintomáticos, através de coagulação com plasma de argônio.

\section{REFERÊNCIAS}

1. AL-HAMID H. Angiodysplasia of the Colon. May 08, 2019.

2. BAUDITZ J, et al. Thalidomide for treatment of severe intestinal bleeding; 2004; 53:609-612.

3. BOCCARDO P, et al. Platelet dysfunction in renal failure. Seminars in thrombosis and hemostasis; $2004 ; 30: 579-89$.

4. DOCHERTY E, et al. Use of small bowel capsule endoscopy in patients with chronic kidney disease: experience from a University Referral Center. Annals of Gastroenterology; 2015, 28, 99-104.

5. FOUTCH PG, REX DK, LIEBERMAN DA. Prevalence and natural history of colonic angiodysplasia among healthy asymptomatic people. Am J Gastroenterology; 1995; 90: 564-7.

6. GALANOPOULOS G. Angiodysplastic Lesions as a Cause of Colonic bleeding in Patients with Chronic Renal Disease: Is There an Association? Saudi J Kidney Dis Transplant; 2012; 23(5):925-928.

7. JACKSON CS, STRONG R. Gastrointestinal Angiodysplasia Diagnosis and Management. Gastrointest Endoscopy Clin N Am 27; 2017; 51-62.

8. KAAROUD H, et al. Gastrointestinal Angiodysplasia in Chronic Renal Failure. Saudi J Kidney Dis Transplant; 2008; 19(5):809-812.

9. KALMAN RS, PEDROSA MC. Evidence-based Review of Gastrointestinal Bleeding in the Chronic Kidney Disease Patient. Seminars in Dialysis; 2014.

10. LEUNG WK, et al. Capsule endoscopy or angiography in patients with acute overt obscure gastrointestinal bleeding: a prospective randomized study with long-term follow-up. Am J Gastroenterology; 2012; 107: 1370-6.

11. MAY A, et al. Long-term outcome after argon plasma coagulation of small-bowel lesions using double-balloon enteroscopy in patients with mid-gastrointestinal bleeding. Endoscopy 2011; 43: 759-765.

12. MIMIDIS K, et al. Thalidomide for Treatment of Bleeding Angiodysplasias during Hemodialysis. Renal Failure, 30:10401041,2008

13. MUFTAH M, et al. Diagnostic and therapeutic considerations for obscure gastrointestinal bleeding in patients with chronic kidney disease. Annals of Gastroenterology;2019; 32,1-11.

14. OLMOS JA, et al. Argon plasma coagulation for prevention of recurrent bleeding from $\mathrm{Gl}$ angiodysplasias. Gastrointest Endosc; 2004; 60: 881-886.

15. SAMI SS, et al. Review article: gastrointestinal angiodysplasia - pathogenesis, diagnosis and management. Aliment Pharmacol Ther; 2014; 39: 15-34.

16. SALTZMAN JR. Angiodysplasia of the gastrointestinal tract. 2018.

17. ZUCKERMAN GR, et al. Upper gastrointestinal bleeding in patients with chronic renal failure. Ann Intern Med; 1985; 102:588.

REAS/EJCH | Vol.Sup.n.48 | e3190 | DOI: https://doi.org/10.25248/reas.e3190.2020 Página 6 de 6 